\title{
MECHATRONIC SYSTEM FOR SOLAR ENERGY ACQUISITION
}

\author{
Besnea Daniel ${ }^{1}$, Dontu Octavian², Gheorghe I. Gheorghe3, \\ Victor Constantin 4 , Spanu Alina ${ }^{5}$ \\ 1,2,4,5 University Politehnica of Bucharest, Mechatronic and Precision Engineering Department \\ Splaiul Independentei Str., 313, District 6 \\ ${ }^{3}$ The National Institute of Research and Development in Mechatronics \\ and Measurement Technique \\ 6-8 Sos. Pantelimon, District 2, Bucharest \\ d bes@yahoo.com,geocefin@yaohoo.com,victor.f.constantin@gmail.com,spanu alina@yahoo.com
}

\begin{abstract}
The paper presents the mechatronic system for solar energy acquisition for using the system fed on. The mechatronic system with two independent movements affords the high accuracy positioning along the two axes for an optic beam narrowing having the main role of focusing the solar energy. The mathematical model and the design of its components are made by using Delphi. The control of mechatronic system with movements along the two axes, which are azimuth and elevation, has been done with microcontroller.
\end{abstract}

Keywords: Solar Energy Locus of Concentration, Mechatronic System, Optical Locus of Concentration.

\section{Introduction}

The solar energy could be transformed into electricity in two ways: directly, by using photovoltaic panels, or indirectly by using the solar energy locus of concentration. The last type of systems are using lens or mirrors and tracking systems for making the focalization of a great area of solar light into a narrow ray. The photovoltaic cells have the role of converting the light into electric energy based on photovoltaic phenomenon.[1].

There are two technologies used for solar energy systems:

- PV - pfotovoltaic systems that use solar panels on the roof or in solar farms on the land, all of them transforming the solar light directly in electrical energy.

- CSP - Systems with concentrated solar power, known as concentrated solar thermal that use the thermal solar energy for steam provider that is transformed into electrical energy later by a turbine.[1]

The working process of photovoltaic panels is without pollution and the direct conversion from solar light into electricity is achieved without any movement. The photovoltaic systems have the main dissadvantage of electric power dependance on received light. Thus, if it is not used an orientation for the system, more than $10-25 \%$ are lost due to the fact that the solar cells are not oriented towards the sun. The dust, clouds and some other contamination sources are reasons for decreasing the output power. This could be compensated by using some other energy sources, such as hydrocarbures.[3]

The photovoltaic modules use the solar energy for generating electricity by photovoltaic phenomenon. Most of them are made of cells put on tiles made of silicon crystalliform. The structure component conduction needed for quantity of electricity is inside the superior or lower layer. The cells should be protected against mechanical actions, wet or some other damaging causes [6].

Some special photovoltaic modules are including beam narrowing that focuses the light by using lens or mirrors on the smaller cells. This fact affords the using of cells with higher cost per unit with higher economic efficiency [6].

\section{Efficiency of Photovoltaic Systems}

Depending on their construction frame, the photovoltaic modules could produce electricity inside a light frequency range, but usually they do not cover the entire solar spectrum (more precisely ultraviolet, infrared and lower strew light).

Consequently, a great solar light quantity is lost during its entrance in module level, so that the efficiency could be increased by using the monochromatic light. The most important idea is the solution of dividing the light in some frequency ranges and send the spot to the special cells designed for these frequencies in order to increase the efficiency with $50 \%$ [6]. 
Nowadays, the bigest efficiency of energy conversion for a single solar module on the market is about 21\%. The most efficient modules manufactured in big series have a power density around $175 \mathrm{~W} / \mathrm{m} 2$ [6].

The conversion efficiency usually about $20 \%$ is diminished by dust, sand or some other particles that are settled out on the solar panels. Their energetic power could be reduced until $30 \%$ in some polluted areas and their cleaning is not an efficient solution [6].

Romania is placed inside an area with better solar energy, with 210 sunny days during an year and having a solar energy flow between 1000 and $1300 \mathrm{kWh} / \mathrm{m} 2$ per year. Considering this entire quantity, we assume that a quantity of 600 and 800 $\mathrm{kWh} / \mathrm{m} 2$ per year is affordable. The most important areas are Black Sea, Dobrogea and Oltenia with a mean value of $1600 \mathrm{kWh} / \mathrm{m} 2$ per year. [7]

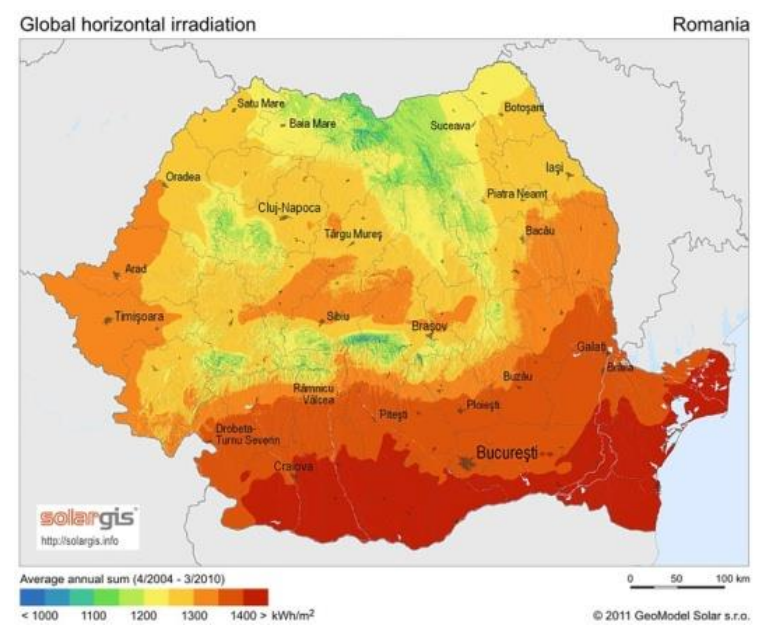

Fig. 1. The sun light for Romania

At the end of 2013 Romania had had an installed capacity of $1151 \mathrm{MW}$ with an increasing of 20 times since 2012. In 2007 the installed capacity was 0.3 MW and it was up to $3.5 \mathrm{MW}$ until the end of 2011 and 6.5 MW until the end of 2012. The maximum value was 1100 MW during 2013, which was an exception, but after that the level decreased at only $69 \mathrm{MW}$ added in 2014. [7]

\section{Tracking Systems}

A solar tracker is a device that gives the sense of direction of an useful load towards the sun. The loads are usually the solar panels, parabolic mirrors, mirrors or lens as well as Fresnel reflector. For plane solar panel, these devices are used for diminishing the incidence angle between the solar rays and photovoltaic panel.

This is increasing the energy quantity produced by a grounded assembly.[8].
For the CPV - Concentrated Photovoltaics and CSP - Concentrated Solar Power applications, these devices assure the right working of optical components these systems are made of. [8]

Even though a grounded panel may acquire in great proportion the light during afternoon, an important energy quantity is available during early morning and later in the afternoon, when a grounded panel could not acquire a needed quantity of energy due to the missalignement. [2][8]

So the main role of such orientation device is to allow the solar energy acquisition for a longer period during the day and with the most favourable alignement with the sun position that is changing during the year, due to the slope of earth axis in front of the sun.

These devices are changing their orientation during the day in order to follow the sun trajectory. The photovoltaic systems are used for minimizing the incidence angle between the acquired light and panel (the angle the light ray makes with the perpendicular direction on the solar panel).

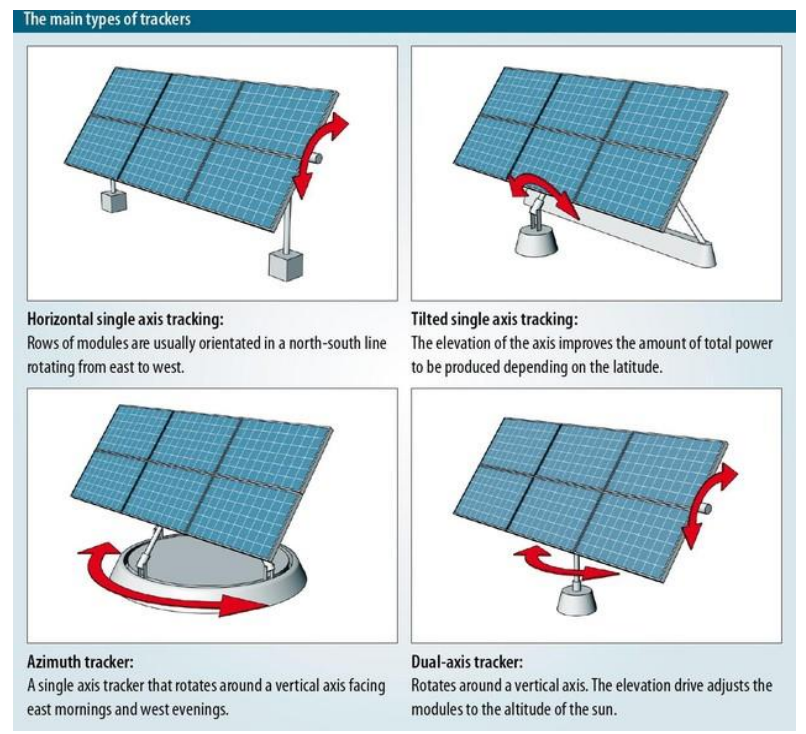

Fig. 2. The types of tracking systems

The Single Axis Trackers have only one degree of freedom, meaning a rotational axis. This is usually aligned along the north meridian, but it could be aligned following any other direction. The horizontal single axis (HSAT) of these trackers is horizontal referenced to the ground. The field assemblies with HSAT are very flexible, their very simple geometry allowing the axes parallelism maintenance for all the panels, which is the only necessary condition for relatively follower of the devices. [8]

The HTSAT - Horizontal Tilted Single Axis Trackers have the modules assembled with an angle for the slope, in comparison with the HSAT where the modules have the horizontal positions.

HTSAT is working on the same principle, maintaining the main axis parallel with the North- 
South direction and moving around the modules from east to west all day long. Generally, these devices are suitable for higher altitude and they do not require a lot of room as the devices with vertical axis, having the advantages of the last one, but beeing cheaper meantime.

The rotational axis of these VSAT - Vertical Single Axis Trackers is vertical with ground reference, so they are moving from east to west during the day. There are more efficient at higher altitude than HSAT. For placing them in the field we have to take into account the possibility of covering them by the other devices nearby, in order to avoid the energy loss and optimizing the useful area.[8]

VSAT have usually the front area tilted with a knowing angle value with the rotational axis. As the module is following the sun trajectory, it is moving around a cone whose axis is coincident with the rotational axis.[8].

The tracker devices with two axes, Dual Axis Trackers have two degrees of freedom, two rotational closing an angle of 900 . The grounded axis with earth reference could be considered primary axis and the other one is secondary.[8]

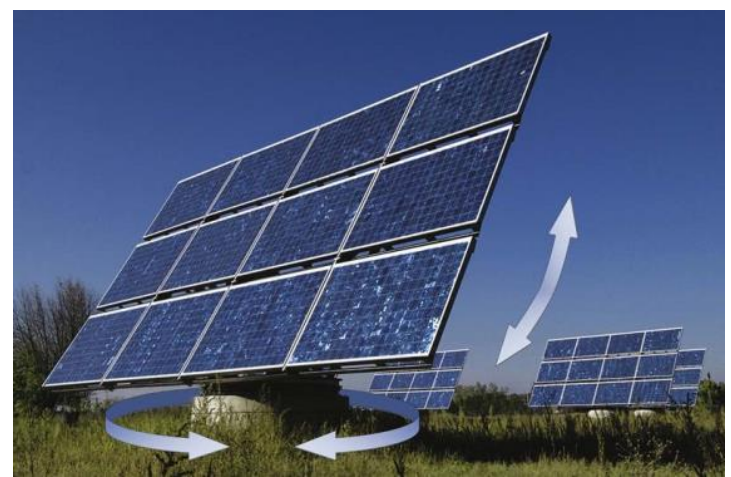

Fig. 3. The rotational axis for a tracker device with two axes.

There are some usually solutions for these devices, classified considering the position of primary axis as earth reference. They have their modules positioned parallel with the secondary rotational axis and the module positions are important for the system performances.

The two axis devices allow the optimal acquisition of solar light due to the possibility of following the solar rays either vertical and horizontal, wherever the sun is, being affordable to be positioned at the demanded angle, in order to be placed directly in front of the sun.

A device with two axes TTDAT - Tip Tilt Dual Axis Tracker has the panel system placed at the top of concrete pillar. The east - west movement is done by the panel system rotating around the pillar.
At the top of bearing is used a $\mathrm{T}$ or $\mathrm{H}$ shape mechanism for rotational movement and for assembling the system. [8][9][10].

The placement of TTDAT are very flexible, the simple geometry allowing the maintenance of parallelism between all rotational axes, which is an optimum way for required positioning of all the tracker devices. It recomended that the devices should be placed with enough room between them, in order to avoid the shadow on the nearby panels when the sun is lower on the sky. TTDAT could compensate that by positioning them with an angle nearby horizontal in order to minimize the shadow, so maximizing the entire energy. [8][9]

The rotational axis of TTDAT are usually aligned along the nord meridian, or along east - west latitude. The devices orientation does not matter, so that they could be placed anywhere it is needed. [8]

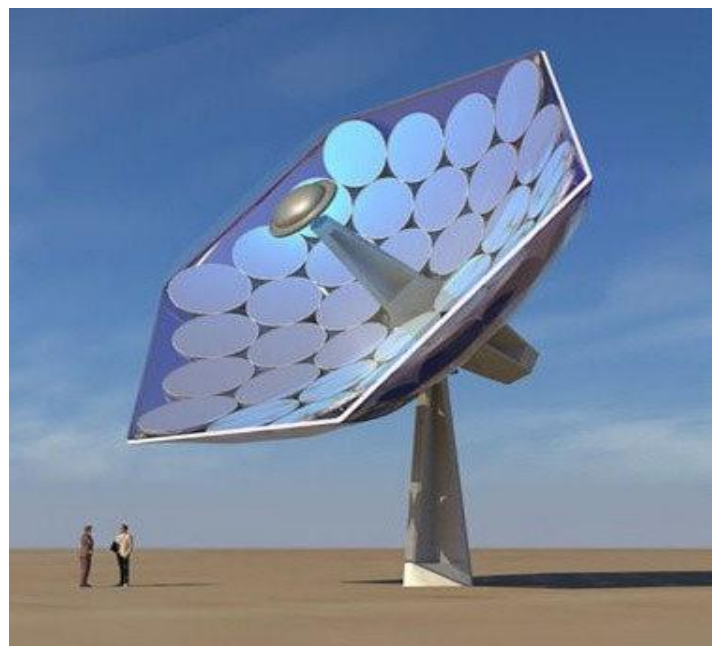

Fig. 4. Photo Voltaics Concentrator (PPV)

The new system PhotoVoltaics Concentrator uses a large number of solar chips water cooled that could transform $80 \%$ of solar radiations into usefull energy. So the system could provide $12 \mathrm{KW}$ of electricity adding $20 \mathrm{KW}$ heating, during a sunny day. Those values are enough for some houses energetical independent with medium level consumption.

\section{The Proposed Designed Solution}

The designed solution is made on the positioning mirror system, the optical system and the electronic system for controlling the electrical stepper motors. For high accuracy positioning, there have been used two electrical stepper motors controlled with Arduino, one for azimuth and the other one for elevation. 


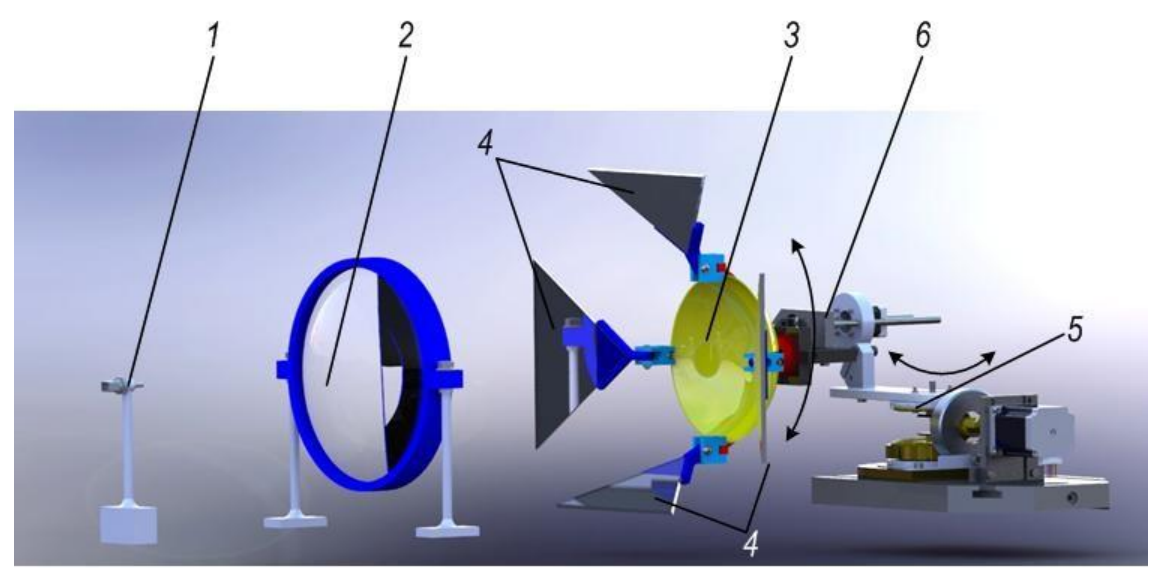

Fig. 5. The CAD model of positioning system and solar energy acquisition: 1 - temperature transducer; 2 - planar convergent lens; 3 - optical focusing; 4 - mirrors; 5 - tracking system for azimuth; 6 - tracking system for elevation

The first system for actuation is made on conical gear used for increasing the mechanical couple, needed for moving the mobile assembly as well for achieving the movement with 90 degrees. The second tracker system on the azimuth is used for transforming the rotational movement of the electrical stepper motor into a translational one, by using a micrometric screw and finally the focusing system axis slope is achieved with an imposed value of angle.

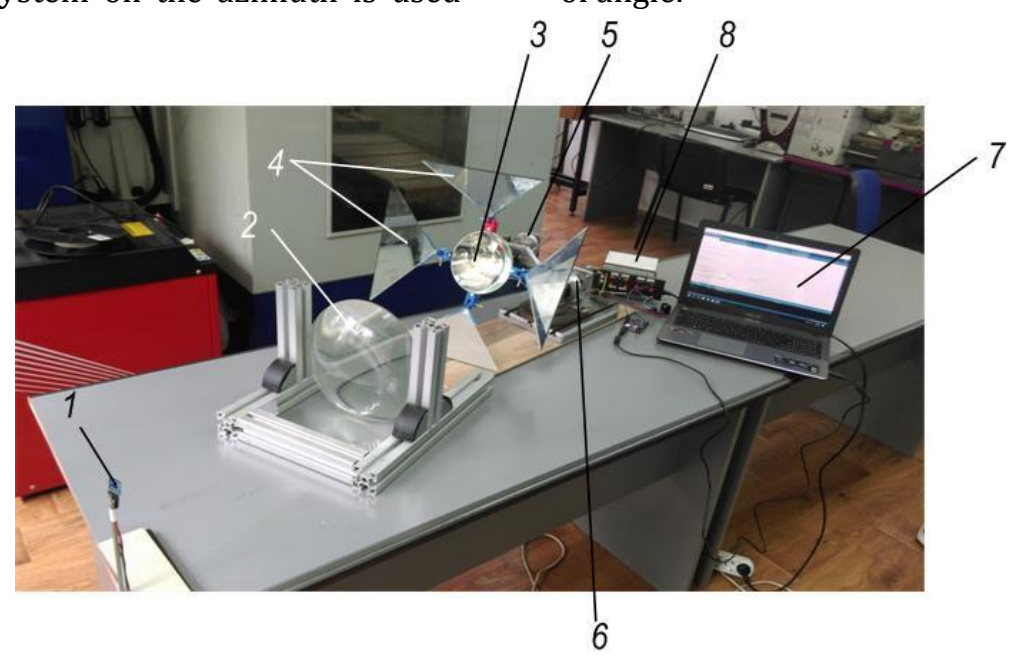

Fig. 6. The positioning system and for solar energy acquisition: 1 - temperature transducer; 2 - plane focusing lens; 3 - optical focusing; 4 - mirrors; 5 - azimuth tracking system; 6 - elevation system tracker; 7 - PC; 8 - electronic system for motor controlling

The designed system is focusing the solar energy on the temperature transducer by using mirrors and plane lens.

The computation of optical parameters and the lens design were made with DELPHY, allowing the very quickly establishing of optical parameters of focusing system.
The design activity is starting with such a lens with the spherical aberration coeficient from aberration of Seidel Theory of third order

$$
Q=A \gamma^{2}-2 B \gamma+C
$$

where: $\gamma=\frac{1}{2}\left(\rho+\rho_{+}\right)$is medium curvature

$$
\begin{array}{ll}
\xi=\frac{1}{\mathrm{~S}} & \text { object proximity } \\
\xi^{\prime}=\xi+\Phi & \text { image proximity } \\
\tau=\frac{1}{2}\left(\xi+\xi^{\prime}\right) & \text { medium proximity }
\end{array}
$$


$\mathrm{A}=\left(1+\frac{2}{\mathrm{n}}\right) \Phi \quad \mathrm{B}=2\left(1+\frac{1}{\mathrm{n}}\right) \Phi \tau \quad \mathrm{C}=\left(3+\frac{2}{\mathrm{n}}\right) \Phi \tau^{2}+\left(\frac{\mathrm{n}}{2 \mathrm{n}-2}\right)^{2} \Phi^{3}$

$\Phi=(n-1)\left(\rho-\rho_{+}\right)$the power of very thin lens

The lens has spherical aberration where the derivative coefficient of spherical aberration as shape coefficient ratio $\gamma$ is null $\frac{\mathrm{dQ}}{\mathrm{d} \gamma}=0$ meaning $\gamma=\frac{\mathrm{B}}{\mathrm{A}}$

If we choose the lens material, we choose the length of radiation ray for designing, the object abscissa and lens diameter, the technological characteristics required by lens manufacturing, and we use the equation of lens power and the definition of curvature. We have obtained two equations as we inferr from eq. 6, where we are explained the curvature resulting the radius of the lens with eq. 1 .

$$
\left\{\begin{array}{l}
\phi=(\mathrm{n}-1)\left(\rho-\rho_{+}\right) \\
\gamma=\frac{1}{2}\left(\rho+\rho_{+}\right)
\end{array}\right.
$$

$$
\begin{cases}\rho=\gamma+\frac{\phi}{2 n-2} & \Rightarrow r=\frac{1}{\rho} \\ \rho_{+}=\gamma-\frac{\phi}{2 n-2} & \Rightarrow r_{+}=\frac{1}{\rho_{+}}\end{cases}
$$

An example of applying these formulas is presented in Figure 1, where on the left side is presented the Table with the computation results, on the central part is presented the lens drawing after the passing from infinite thin lens to the finite thin lens, and on the right side is presented the evolution of spherical aberration as curvature function.

We infer that for the condition of derivative nullification we have a minimum. For the lens material we have imposed the optical glass F5 with the refraction coefficient for $d$ radiation $n=1.60342$ with dispersion $v=38.03$.

After computing the eq 1-7, we have to choose the coefficient for applying finite thickness $\mathrm{k}=$ 0.02 .Finally, we have obtained the values presented in Fig.8, where there are the axis parallel characteristics too.

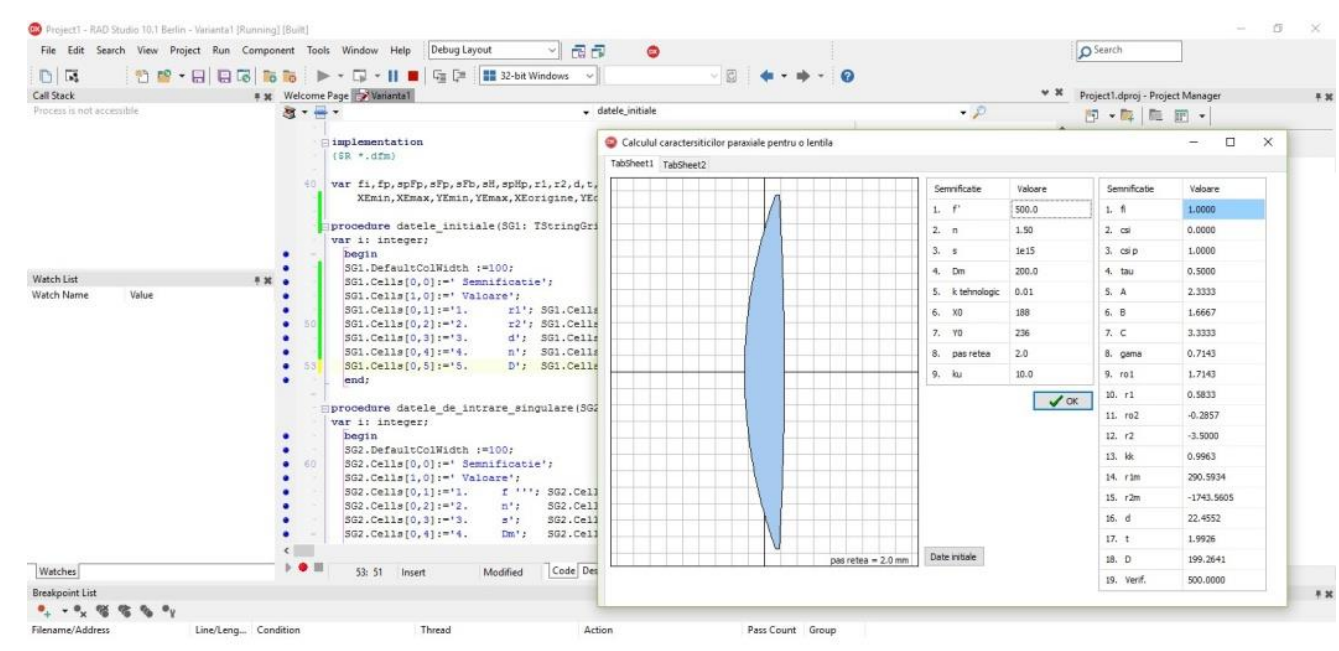

Fig. 7. The calculus of axis parallel characteristics for the lens by using the Delphi software

\section{Conclusions}

The working process of photovoltaic panels is done without any pollution and the direct conversion of sun light into electricity has a lot of advantages.
The main dissadvantage is the dependence of electric power on the direct sun light.

So, if a tracking system is not used a $10-25 \%$ is lost due to the missalignement of sun light cell with the sun rays. 
The dust, the clouds and the sand inside the athmotsphere are some other factors that are disturbing the process. All of these could be improved by using some other complementar energy sources, such as hydrocarbon. [3]

The using of tracking devices could increases the efficiency of the electric energy provider about $0.33 \%$ comparing with the grounded modules working with a knowing angle. Considering any solar application, we may say that the conversion efficiency could be improved by continous control of optimum angle during the sun movement all day long. Due to the fact that the solar efficiency of conversion implies the process efficiency increasing, by using the tracking devices, a huge amount of power could be realized if a big system is used. [11][12]

There are some dissadvantages of tracking devices. If we use it, we add some suplementary equipments, some moving systems and gears, which will demand regular service. Moreover, if the tracking system is broken when the solar panels are oriented at the bigest angle, the power losses will be great until the system will work again. This is the reason why the tracking devices will be more easily damaged by the storm. [11]

\section{References}

[1] „Solar Power”, En.Wikipedia.Org, https://en.wikipedia.org/wiki/Solar_power.

[2] Reed Crossley, „What's the Difference Between Single and Dual-Axis Solar Trackers?". Revolve Solar | Solar Installers in Austin and Northern California,

https://www.revolvesolar.com/whats-thedifference-between-single-axis-and-dual-axissolar-trackers.
[3] „Photovoltaics”, $\quad$ En.Wikipedia.Org, https://en.wikipedia.org/wiki/Photovoltaics.

[4] „Photovoltaic Effect”, En.Wikipedia.Org, https://en.wikipedia.org/wiki/Photovoltaic eff ect.

[5] „Solar Cell”, En.Wikipedia.Org, access: https://en.wikipedia.org/wiki/Solar_cell

[6] „Solar Panel”, En.Wikipedia.Org, https://en.wikipedia.org/wiki/Solar_panel.

[7] „Solar Power in Romania”, En.Wikipedia.Org, https://en.wikipedia.org/wiki/Solar_power_in_ Romania.

[8] „Solar Tracker”, En.Wikipedia.Org, https://en.wikipedia.org/wiki/Solar_tracker.

[9] „Solar Tracker Market Expected To Grow । Evolve India", Evolveindia.In, http://evolveindia.in/blog/2015/10/24/solartracker-market-expected-to-grow/.

[10] „Reading Article: Follow the Sun: Reliable Positioning for Solar Tracking Systems, Written by Fraba Posita", Energetica-International.Com, http://www.energeticainternational.com/articles/follow-the-sunreliable-positioning-for-solar-tracking-systems.

[11] Zipp, K.: „What Is a Solar Tracker?”, Solar Power World, http://www.solarpowerworldonline.com/2013 /04/how-does-a-solar-tracker-work/.

[12] „Heathrow Solar Energy | Heathrow Energy Solutions | Heathrow Energy Independence", Usnovacorp.Com,http://www.usnovacorp.com/ available-technologies.php.

[13] Bacescu, D.:"Optica Aplica. Analiza si sinteza componentelor". Editura Medra. Bucuresti. 2004. 